\title{
Has the EAGLE landed for the use of clear lens extraction in angle- closure glaucoma? And how should primary angle-closure suspects be treated?
}

\author{
Luke Tanner ${ }^{1} \cdot$ Gus Gazzard $\mathbb{C}^{2,3} \cdot$ Winifred P. Nolan ${ }^{2,4} \cdot$ Paul J. Foster $\mathbb{C}^{2,3,4}$
}

Received: 1 July 2019 / Revised: 9 September 2019 / Accepted: 10 September 2019 / Published online: 24 October 2019

(c) The Author(s), under exclusive licence to The Royal College of Ophthalmologists 2019

\begin{abstract}
Angle-closure glaucoma is an aggressive condition that causes millions to become blind worldwide. This review explores the use of prophylactic laser peripheral iridotomy (PI) in patients classified as primary angle-closure suspects (PACS), and additionally, the use of clear lens exchange as a primary treatment option in established angle-closure disease with or without glaucoma. As PI has a strong prophylactic effect in fellow eyes of patients who have had an acute attack, its use has been widely adopted in those patients classified as PACS, but with limited evidence to support this. A large randomised trial conducted in China has demonstrated that although PI reduces the risk of incident angle-closure disease, the incidence of disease that would threaten vision was much lower than anticipated. This suggests that the benefit of prophylactic PI is very limited. Health services data shows an association between rising cataract surgical rates and of decreasing rates of acute angle-closure. Age-related growth of the lens is a major component of angle-closure disease. Several studies have shown that clear lens extraction (CLE) effectively lowers IOP in angle-closure. The use of CLE as a primary treatment option has been tested against LPI in the EAGLE study, a large RCT that enroled people with angle-closure and an IOP $>30 \mathrm{mmHg}$, and those with angle-closure glaucoma. The trial showed CLE to be superior to PI both for IOP control and patient reported quality of life. On these grounds, CLE should be considered for first-line treatment of more advanced angle-closure disease.
\end{abstract}

\section{Introduction}

Glaucoma is a common neuropathy in which there is an excavated atrophy of the optic nerve head and progressive loss of vision, typically starting 10 to 20 degrees from fixation. It is associated with increased intraocular pressure (IOP), although many cases develop with IOP remaining in the statistically normal range [1]. It is the most common neurodegenerative condition worldwide and is the second

Paul J. Foster

p.foster@ucl.ac.uk

1 University of Exeter Medical School, College of Medicine \& Health, St. Luke's Campus, Heavitree Road, Exeter EX1 2LU, UK

2 Glaucoma Service, Moorfields Eye Hospital, City Road, London EC1V 2PD, UK

3 UCL Institute of Ophthalmology, 11-43 Bath Street, London EC1V 9EL, UK

4 NIHR Biomedical Research Centre at Moorfields Eye Hospital \& UCL Institute of Ophthalmology, London EC1V 2PD, UK biggest cause of blindness [2]. Angle-closure glaucoma accounts for $25 \%$ of cases and is estimated to affect 20 million people, with $75 \%$ of those affected living in Asia [3, 4]. Angle-closure occurs when the anterior chamber angle becomes occluded by the iris, reducing the drainage of aqueous humour through the trabecular meshwork (TM), which consequently increases the IOP. It can be as a result of several factors, including a relatively thicker and more anteriorly positioned crystalline lens, a thicker anteriorly displaced and more anteriorly inserted iris and an anteriorly positioned ciliary body and its processes and the degree of pupil block [5]. Angle-closure can be further classified according to the natural history of the condition into primary angle-closure glaucoma (PACG), primary angleclosure (PAC) and primary angle-closure suspect (PACS). PACG is usually defined as at least $180^{\circ}$ degrees of iridotrabecular touch with glaucomatous optic damage. PAC has the same degree of iridotrabecular contact with high IOP but without glaucomatous damage. PACS is defined as the same level of iridotrabecular contact but with normal IOP and no signs of glaucomatous optic neuropathy [6]. This review focuses on the current treatment options for 
patients with primary angle-closure, with or without glaucoma. The role of lens extraction as a primary treatment option is explored, and in addition, the role for prophylactic laser iridotomy in those patients with PACS is evaluated.

\section{Changes in management of primary angle- closure}

In 1856, Albrecht von Graeffe described the use of surgical peripheral iridectomy in "acute glaucoma", reporting it to be successful in treating many eyes, which suddenly became "stony hard". The procedure evolved in the 1970's with the introduction first of the argon laser, and again in the 1980's with the advent of Nd:YAG laser [7, 8]. Laser iridotomy has now become established as the first-line intervention for primary angle-closure, both as a treatment in acute, symptomatic cases, as well as in chronic, asymptomatic angleclosure. There is strong consensus that a laser iridotomy or surgical iridectomy is indicated in the fellow eye of people who have presented with an acute attack, and that it should be attempted in the eye suffering the acute pressure rise once symptoms and corneal clarity permit $[9,10]$. The role of laser iridotomy in the management of chronic, asymptomatic angle-closure has been subject to increasing scrutiny, with the current evidence for benefit appearing weaker than has long been believed. At the same time, there has been a growing body of evidence supporting the use of lens extraction for the management of primary angle-closure. Greve proposed the use of extracapusular cataract extraction as a viable option for primary angle-closure glaucoma, and later suggested that this technique should be considered even in eyes with "good visual acuity" [11, 12]. Greve and Gunning went further, questioning the paradigm of trabeculectomy as the cardinal surgical option for eyes with uncontrolled intraocular pressure, stating: "Drainage surgery in patients with angle-closure glaucoma proved to be associated with multiple surgical interventions and deterioration in visual function. The choice of first a cataract procedure with the option of a future trabeculectomy may be a more attractive approach in patients with subacute or chronic angle-closure glaucoma than trabeculectomy followed by an optional cataract procedure" [13]. Many others have continued to examine the effect of cataract surgery on primary angle-closure, with encouraging results [14-16].

Health services activity data have been used to explore the impact of various interventions on the rate of angleclosure disease at a population level. Data spanning an 8year period, drawn from the Taiwan national health database, showed a reduction in admissions of patients with acute primary angle-closure occurring in conjunction with a rise in cataract surgery across the east Asian nation [17].
UK hospital episode statistics (HES) data were used to probe the same question, with a similar conclusion-that the frequency of admissions with a diagnosis of angleclosure had declined significantly between 1999 and 2004, while cataract surgical rates had increased markedly [18]. One of us (PJF) suggested that the use of laser iridotomy had also increased over this period in the UK, and that this may explain the decline in angle-closure admissions [19]. However, this theory is probably inaccurate; Colleagues used Scottish health services activity data (ISD-Information Services Division, Scotland) to examine the rate of angle-closure presentations, cataract surgery and laser iridotomy, showing that the rise in cataract surgery clearly mirrors a decline in angle-closure episodes, while the rise in laser iridotomy procedures only appears once the decline in angle-closure is very well established [20]. Against this backdrop, a series of randomised clinical trials carried out over the last two decades now provides evidence that guide and inform the care of patients with, or at risk of, angleclosure glaucoma. The fact that these trials have used the natural history staging system described above enhances the comparability of the results, and form the framework for diagnosis and management of angle-closure and its related conditions.

\section{Laser peripheral Iridotomy (LPI) as a prophylactic treatment in those who are PACS}

Angle-closure glaucoma causes millions of people to become blind worldwide, making it a potential target for preventive public health policy. There are clear biometric risk factors that can identify those at risk with reasonable precision [21-23]. The strong prophylactic effect of iridotomy and iridectomy in the fellow eye of people who suffer acute angle-closure suggests that peripheral iridotomy (PI) could be used more widely in preventing primary angleclosure glaucoma. The number of those at risk (PACS)s is high, with 28 million individuals in China alone [4]. Against this backdrop, laser PI has become widely used as a prophylactic treatment for these people. The belief in the efficacy of this strategy is underlined by the fact that $75 \%$ of UK consultant ophthalmologists offer prophylactic laser PI to their patients [24]. Despite this, the evidence is limited.

\section{The risk of angle-closure in people with narrow angles}

The incidence of acute presentations with angle-closure is low and declining $[17,18,20]$. It is reasonable to assume this is a proxy measure for asymptomatic disease, although 
this assumption has never been formally addressed in research, and is therefore unproven. Rates of acute angleclosure in the generally white population of Europe is between 2 to 7 cases/100,000 people per year in those aged over 40 years, and 2/100,000/year in the overall population [25-29]. Among Asian people, the rates are in the region of 6/100,000/year in Singaporean Indian and Malay (south East Asian) people aged 30 years and older [30]. Chinese people are at highest risk with incidence rates of around 12 to $15 / 100,000 /$ year $[30,31]$.

The risk of developing significant disease in people deemed primary angle-closure suspects has always been presumed to be relatively high. A study in the United States prospectively examined 129 patients thought to be at risk. These people were then followed up with no treatment. Mean follow-up was 2.7 years with a range up to 6 years. Twenty-five patients developed angle-closure in at least one eye during the follow-up period. An important finding that has generally escaped most readers was that, in most (17 of the 25 patients), the angle closure was nonacute (there were no clinical signs or symptoms and no increase in intraocular pressure). While chronic, asymptomatic angle-closure is a well-recognised clinical characteristic among Asians, it is a less well-recognised presentation among white people of European origin. None of the tests carried out at baseline gave a high sensitivity or positive predictive value for detecting the eyes that later developed angle closure [32].

A pair of parallel studies in Vellore, southern India, enroled participants in a community setting and examined the incidence of angle-closure disease among suspects, and angle-closure glaucoma in those with angle-closure disease. Each group were studied for 5 years. The incidence of disease among suspects (new cases of PAC in those with PACS) was $22 \%, 95 \%$ CI: 9.8 to $34.2 \%$ (50 of the 82 PACS cases examined) over 5 years, or $\sim 5.5 \%$ per year. Among the 11 cases of PAC disease, seven had synechial disease and four appositional; at baseline, all were bilateral PACS. One person among the 110 normals progressed to PAC. There was no significant difference in axial length (AL), anterior chamber depth or lens thickness between those who progressed and those who did not. None of the patients developed optic disc or field damage attributable to angle closure [33]. Among the 37 patients diagnosed with PAC disease at baseline, 28 of 32 PAC subjects who could be contacted attended for examination. Eight $(28.5 \%$; $95 \%$ CI $12-45 \%$ ) had progressed to PACG over the 5-year followup period; two of seven with appositional and six of 21 with synechial closure. All were advised to undergo laser peripheral iridotomy (PI) in 1995; one of the nine who underwent LPI progressed compared to seven of 19 who refused LPI. Again, there was no significant difference in biometric parameters between those who progressed and those who did not. In common with the US study none developed acute angle-closure, and none became blind due to glaucoma [32, 33].

\section{Prophylactic peripheral laser iridotomy}

Epidemiological research in Mongolia between 1995 and 2000 documented a high prevalence of primary angle-closure, identified potential screening tests, and recorded risks and short-term benefits of laser iridotomy [21, 22, 34, 35]. A decision was made to proceed with a randomised trial of screening in 1999 in Mongolia. This study allocated a group of people aged over 50 to either a control group or an intervention group, in which the intervention under test was screening and prophylactic treatment (not solely the treatment). The unit of randomisation was at the person level. Participants were screened for occludable angles and if present, offered LPI [36]. At 6 years follow-up they found no benefit in the prevention of PACG between groups, suggesting that screening and prophylactic LPI may not be efficacious. A major consideration with this study was the considerable loss of follow-up [37].

However, the primary focus for tackling angle-closure glaucoma as a public health concern was, and probably will always be, China, with a population of over 1 billion people. Using data from Mongolia and Singapore, it has been estimated that, in 2000, 9.4 million people aged 40 years and older in China had glaucomatous optic neuropathy. Of this number, 5.2 million $(55 \%)$ would be blind in at least one eye and 1.7 million $(18.1 \%$ ) were blind in both eyes. Primary angle-closure glaucoma (PACG) was likely responsible for the vast majority $(91 \%)$ of bilateral glaucoma blindness in China at the time. The number of people with the anatomical trait predisposing to PACG (an "occludable" drainage angle) would be in the region of 28.2 million, and of these, 9.1 million would have significant angle closure, indicated by peripheral anterior synechiae or raised intraocular pressure [4]. Further population-based research in Guangzhou, in southern China, identified a prevalence of PACG of $1.5 \%$ (95\% CI: 0.8 to $2.1 \%$ ) in people over the age of 50 years. In this population, $10 \%$ had a drainage angle configuration that would make them primary angle-closure suspects (PACS) [38, 39]. Research carried out in parallel with these studies of disease prevalence demonstrated a significant increase in the angle width in Chinese people with narrow angles after laser PI. The authors concluded that long-term prospective studies with a larger sample size are required to determine if the risks of PAC glaucoma and other related pathologic sequelae are reduced after prophylactic LPI, and that there was a need to investigate the risk-to-benefit ratio before recommending widespread use of prophylactic LPI in this population [40]. 
The ensuing large randomised controlled trial (ZAPThe Zhongshan Angle-closure Prophylaxis Study) conducted by $\mathrm{He}$ et al. aimed to provide the first robust evidence on whether there was a benefit from offering prophylactic LPI in a high-risk population. The study randomised treatment by eye, leaving one eye per participant untreated as an age, sex, and biometrically matched control. This was a significant difference from the randomised trial of screening carried out in Mongolia, as the question asked in the Chinese trial was simply about the benefit of the treatment, not the overall screening package [37]. The study took place in Guangzhou city in Southern China, where researchers screened 11,991 people aged 50-70-years-old, aiming to identify all bilateral PACSs. Eight-hundred eighty-nine participants were enroled and each received LPI to one randomly selected eye, with the contralateral eye serving as a control. During the trial, it became apparent that the event rate was much lower than predicted from existing studies of disease incidence. For this reason, a second round of recruitment was carried out and the follow-up period lengthened from 36 to 72 months. The primary outcome was the incidence of primary angle-closure disease, manifesting as either raised IOP, new peripheral anterior synechiae or an acute episode of symptomatically raised IOP. The study found the primary outcome (PAC) incidence as 4.19 per 1000 eye years (19 eyes) in the treatment group and an incidence of 7.97 per 1000 eye years ( 36 eyes) in the control group, which was a statistically significant difference. This meant that the LPI group had a $47 \%$ risk reduction in developing PAC. While this was a statistically significant reduction in risk ( $p=0.0041)$, prophylactic laser PI treatment did not result in a dramatic reduction of disease risk in the population, as the incidence of angle-closure disease (the rate of newly occurring disease) with no treatment was less than $1 \%$ per year [41]. Furthermore, "endpoint" cases were relatively mild presentation. Of the 55 people who developed new disease in the trial, only five suffered an acute attack, with three cases being secondary to pupil dilation during the course of investigations carried out under trial protocol. Two people suffered a spontaneous acute attack in the untreated eye. Overall, there were three acute episodes in untreated, control eyes and two in treated eyes. The major disease feature identified as an endpoint was new peripheral anterior synechiae (PAS), occurring in 15 treated and 30 untreated eyes. The trial concluded that the vast majority of those reaching an endpoint were at low risk of significant loss of vision in the foreseeable future [41].

There were 24 control eyes (randomised to no treatment) that received LPI outside of the trial protocol and follow-up. This may have biased the results as it is possible that, without the laser treatment, these patients could have gone on to develop the incident disease. This could have increased the overall rate of disease and increased the difference in outcome between the treatment groups. Another factor potentially influencing the results is that around half (54.8\%) of Chinese patients with PACG have a mixed mechanism [40, 42, 43]. As LPI is most effective at treating pupillary block mechanism specifically, it may explain why its performance in the study, where all the subjects were Chinese, was considered modest [42]. Further supporting this, Asian patients' angle closure persists even after LPI in $19 \%$ [40]. Ten people in the trial met the outcome in both eyes, highlighting that in these cases, LPI offered no benefit compared to no treatment. The study found that no serious adverse events occurred with LPI, supporting that it is a safe intervention [41].

The primary question addressed by the ZAP trial was that of the benefit of preventive laser iridotomy in PACS detected in a screening programme. The study population included some of the highest risk individuals worldwide. The untreated control eyes provide an insight into the natural history of primary angle-closure. The rate of conversion from PACS to angle-closure disease was very low. While treatment halved the risk of conversion, in overall terms, the benefit provided by LPI was modest. Considering the utilisation of scarce resources, and of opportunity cost, the number "needed to treat" (NNT) concept is helpful. This is calculated as the reciprocal of the absolute risk reduction. The annual risk reduction for primary angle-closure disease was $0.38 \%$, meaning that 44 patients would need to be treated to prevent one new case of primary angle disease in 6 years. Assuming that these primary angle-closure cases have a $35 \%$ risk of developing sight loss from glaucoma over a further 5 years, and assuming that prevention of sight loss would be the ultimate goal of prophylactic laser iridotomy, then it would be necessary to treat around 126 people to prevent one new case of sight loss from glaucoma in a decade. The cost utility value of prophylactic PI has yet to be determined. However, this high NNT might make laser peripheral iridotomy non-viable as a strategy for preventing loss of vision in socialised medicine systems or in health insurance systems, where other health interventions might be superior in terms of benefits and lower in cost. Efforts to identify PACS and treat with iridotomy on a population basis probably are not the best use of resources, and health-care systems would be more effective if they allocated resources to identifying glaucoma earlier. The authors of the ZAP study are running a sister randomised control trial (RCT) in Singapore, which has yet to publish results in a peer-reviewed journal. However, results have been presented at ARVO (The Association for Research in Vision and Ophthalmology) showing a similar halving of angle-closure incidence in eyes that were treated with prophylactic laser iridotomy. The incidence of disease 
(PAC) was around 2\% per annum in untreated eyes, and $1 \%$ in the treated eyes, although the precision of these figures has yet to be calculated. However, the current evidence from the trial of screening in Mongolia, and the randomised trial of prophylactic treatment in Guangzhou, China (both high-risk populations) suggest that screening for PACS and offering prophylactic treatment is of very limited benefit, and unlikely to be cost-effective. One must also consider the external validity. As these studies were conducted solely in Asian people in whom non-pupil block mechanisms seem to play a greater role inferring results apply to the UK population is subject to some uncertainty [44]. The ZAP trial was not able to identify risk factors that might identify a particularly susceptible group on whom treatment could be targeted. Taking all evidence on the frequency of angle-closure in the population at large, and the effect of prophylactic laser iridotomy, there are two conclusions that can be drawn. Firstly, laser iridotomy halves the risk of incident angleclosure disease in those people at highest risk. Secondly, the rate at which new angle-closure disease manifests is much lower than previously supposed, meaning the benefit from prophylactic PI in PACS is very small. It currently appears that there is minimal benefit from either structured or opportunistic screening and prophylaxis in this condition.

Laser iridotomy should continue to be discussed and offered in the very highest risk PACS eyes among vulnerable groups such as:

- Fellow eyes of those that have suffered acute angleclosure crises [45].

- Need for regular pharmacological mydriasis for retinal diagnosis or monitoring [29].

- The use of tricyclic or SSRI antidepressant medication [46, 47].

- A family history of glaucoma [48].

- People who live or work in remote areas with limited access to care, such as active duty armed forces, humanitarian aid workers, engineers on oil rigs etc.

Additionally, there is some suggestion that cold and flu medication containing strong nasal decongestants may increase risk, although it is unclear if this is association or causation [49-51]. People who make regular, long-haul air journeys, and those who live or work in remote regions of the world where emergency ophthalmic care is not available may be reassured by undergoing prophylactic laser iridotomy [52]. However, until evidence becomes available to the contrary, the widespread practice of identifying people with narrow angles and no other risk factors, and encouraging laser iridotomy is not supported by evidence.

\section{To dilate or not to dilate?}

There is often anxiety among clinicians about the risks of dilating the pupil in situations where it facilitates urgent care, such as when a retinal detachment is suspected, or when confirmed, vitreoretinal surgery is needed. The risks of acute angle-closure after dilation are low, in the order of $3 / 10,000$ [53]. Expert opinion is that the benefits of confirming a diagnosis, and timely delivery of treatment, outweigh the risks [54]. The predilation IOP and a known history of glaucoma are risk factors for a postdilation IOP $\geq 25 \mathrm{mmHg}$, and these factors should be assessed prior to dilation in all cases. If dilation is required for diagnosis or treatment, this should go ahead unless the IOP is $\geq 24$ $\mathrm{mmHg}$, in which case, a cause should be sought and addressed prior to dilation. In such cases, an opinion from a glaucoma specialist should be sought in a timely fashion after dilation. The indication for laser iridotomy is the same as used in the trial outlined above- that of a gonioscopic finding of $>180$ degrees of iridotrabecular contact. If a pressure rise is detected, initial management with oral or intravenous acetazolamide (excluding those with known allergies) is the preferred option. The use of pilocarpine is not appropriate, as it may splint the pupil in a mid-dilated position, in effect, creating a situation similar to that in a Mapstone provocative test $[55,56]$. In a population survey in Singapore, all participants were dilated, and those with occluded angles were give oral acetazolamide $250 \mathrm{mg}$ on leaving the clinic, and a further $250 \mathrm{mg}$ at bed time the same day. None reported symptoms indicating an IOP rise when contacted by phone the following day [57].

\section{Lens extraction as a treatment for angle- closure}

Age-related growth of the lens is a major contributing factor in the development of PACG and for patients with coexisting cataract, lens extraction is an established management option [58]. Melese et al. [59] used anterior optical coherence tomography to measure the angle parameters in patients before and after cataract extraction, comparing these to LPI. The study found the angle width parameters were significantly increased following cataract extraction compared to LPI. A randomised trial by Lam et al. [60] examined 62 Chinese cataract patients who also had suffered acute PAC, found that early phacoemulsification was more effective at preventing a future rise in IOP than was LPI. Another RCT conducted by Husain et al. [61] allocated 37 participants to either LPI or phacoemulsification for patients with early acute angle closure with co-existing cataract. The results echo those of Lam et al. by also demonstrating a lower rate of IOP failure in the 
phacoemulsification group at 2 years. A meta-analysis conducted by Masis et al. [62] also found that clear lens extraction (CLE) in patients with PACG lowered the IOP by $-6.4 \mathrm{mmHg}(95 \% \mathrm{CI}:-9.4$ to -3.4$)$ at final follow-up. A small prospective case series involving 44 eyes, carried out in India by Dada et al. [63] evaluated the effect of CLE on patients who had PAC. The study looked at patients who still had a raised IOP $(>25.0 \mathrm{mmHg}) 8$ weeks after LPI, despite ocular hypotensive medications. Success was defined by an IOP $<18 \mathrm{mmHg}$ without medication, this was reached by $86 \%$ with the remaining $14 \%$ requiring only one medication to achieve the same IOP. The study concluded that CLE resulted in a significant reduction in IOP, a reduced need for medication and a significant increase in anterior angle parameters. A significant negative correlation was also found between lens thickness and anterior chamber depth, further supporting the rationale for CLE in widening the anterior chamber angle.

A randomised trial by Tham et al. [64] compared cataract extraction with and without trabeculectomy in 72 patients with chronic PACG who were medically uncontrolled. The study found that phacoemulsification with trabeculectomy was a marginally more effective option at lowering IOP compared to phacoemulsification alone, although this difference was not significant or clinically relevant. A possible explanation to this could be the increased scarring present in the angle of patients with chronic angle-closure where phacoemulsification alone may be less effective. In addition, phacotrabeculectomy was found to have a higher complication rate [64]. The same author also completed a retrospective analysis of two RCTs to determine if there are any clinical factors relating to a failure to control IOP post phacoemulsification or phacotrabeculectomy in PACG. Failure was defined as an IOP of $21 \mathrm{mmHg}$ or greater or requiring glaucoma drugs to maintain an IOP $<21 \mathrm{mmHg}$ at the 24-month follow-up. The study identified the following factors: a higher preoperative IOP [odds ratio (OR) 1.7 per increase in IOP of 5 $\mathrm{mmHg}$, a greater preoperative requirement for glaucoma drugs (OR, 1.9), and phacoemulsification alone (OR, 10.2) [65]. However, the confidence intervals for phacoemulsification alone are very wide indicating that the OR may be higher or lower. Due to the high risk and rate of complications with trabeculectomy (including hypotony), and the fact that the failure rate is higher in acutely inflamed eyes, this data suggest its use should be reserved for later in the treatment pathway.

As outlined previously, there is clear evidence supporting the use of lens extraction for patients with angle-closure. The procedure consistently widens the angle and lowers IOP. However, CLE as a primary treatment option is not as widely practiced as is LPI, which is entrenched in angleclosure treatment guidelines. The EAGLE study, a large multicentred RCT funded by the UK's Medical Research Council (MRC), involving 419 patients assessed the efficacy, safety and cost-effectiveness of CLE vs. LPI (plus medication), as a primary intervention. Measured outcomes included validated questionnaires, to obtain information on quality of life of patients in both groups to assess the efficacy and to calculate the Quality-Adjusted Life Years (QALY), necessary for the cost utility analysis. IOP was measured by a masked observer over 36 months post randomisation. The study found no change in patient reported quality of life (EQ5D questionnaires) and a significant lowering of IOP $(-1.2 \mathrm{mmHg})$ over 3 years of follow-up in the CLE group. It also found a reduction in the need for further medications and surgeries in the CLE group. Quality of life scores deteriorated in the standard treatment group (laser PI), but remained stable in those undergoing CLE. The stability in health-related quality of life questionnaire scores for CLE could be attributed to the reduced future need for medication and surgeries [66].

Assessing the cost-effectiveness, CLE gave an increased mean QALYs score at 3 years and while the initial cost is higher, the study found that the cost would likely be within the ceiling willingness-to-pay ratio of the National Health Service (NHS). In addition, the incremental cost ratio for CLE can be partially offset by reduced follow-up, medications and further surgeries.

A detailed supplementary analysis of costs of primary and secondary health-care usage from the UK NHS perspective, examining quality-adjusted life years (QALYs) and the incremental cost-effectiveness ratio (ICER) for lens extraction vs. standard care, found mean health service costs were higher in patients randomised to lens extraction: $£ 2467$ vs. £1486. The mean adjusted QALYs were also higher with early lens extraction: 2.602 vs. 2.533 . The ICER for lens extraction vs. standard care was $£ 14,284$ per QALY gained at three years. Modelling suggests that the ICER may drop to $£ 7090$ per QALY gained by 5 years . The authors concluded that CLE had a 67-89\% chance of being cost-effective at 3 years and that it may be cost saving by 10 years [66].

This study has provided compelling evidence to support lens extractions as a first-line treatment for patients matching the study enrolment criteria. However, it is important to remember that the results do not directly inform the care of those with other more or less severe features of disease who were not enroled. Younger patients who can still accommodate and those PAC patients with modestly raised IOP (under $30 \mathrm{mmHg}$ ) were not included, so the benefits for these patients remain unproven. While this study shows equal rates of surgical complication in both groups, the severe sight threatening complication associated with CLE must be considered by individual patients [66]. Lens extraction is not currently justifiable for management 
of narrow drainage angles without other pathology or significant risk factors.

Phacoemulsification of the lens with intraocular lens implant is a low-risk procedure. One meta-analysis looking at complication rates of cataract surgery found only $2.23 \%$ had sight impacting complications [67]. However, the rates of complications in patients with PACG must be considered; the AL of the eye is often shorter, the anterior chamber more shallow and the IOP higher, resulting in a more technically challenging surgery. One retrospective study assessing clinical outcomes in patients with PAC undergoing cataract surgery, by Shams et al. [68] reported their complication rates. They reported a complication rate of $12.7 \%$, which included: anterior capsular tear, clinical cystoid macular oedema, anterior uveitis and early rise in IOP $>22 \mathrm{mmHg}$. No major complications (aqueous misdirection or uveal effusion syndrome) were reported. Another study by Day et al. [69] evaluated clinical outcomes in nanophthalmic eyes undergoing phacoemulsification, which are at high risk of developing PACG. The complications identified in this study help evaluate the potential added risks to CLE in angle closure. The study looked at 103 eyes, all with an ALs of $<21.0 \mathrm{~mm}$ and found complications to occur in 16 cases $(15.5 \%)$. Intraoperative complications occurred in six cases (5.8\%). Five patients had intraoperative zonular dehiscence and the in other case, the intraocular lens (IOL) broke on unfolding. Postoperative complications occurred in 13 eyes $(12.6 \%)$, four cases had severe postoperative uveitis, which resolved after intensive topical steroid treatment, seven eyes had uncontrolled IOP due to aqueous misdirection and two eye had an IOL exchange. The study found shorter AL and an IOP of more than $22 \mathrm{mmHg}$ to be independent risk factors for complications. On sub-analysis of $\mathrm{AL}$, an $\mathrm{AL}$ of $<20.5 \mathrm{~mm}$ was associated with a four times higher odds of complication, whereas an $\mathrm{AL}$ of $<19.0 \mathrm{~mm}$ resulted in a 21 times higher odds. This further highlights the risks of surgery in abnormally small eyes. The study concluded that while surgery is technically challenging in nanophthalmic eyes, it was safe with reported complications less than in previous literature, especially when comparing phacoemulsification with trabeculectomy. However, the surgeons in the study were experienced at managing these difficult surgeries, important when considering whether CLE is safe as primary treatment for chronic angle-closure disease. The EAGLE study also published the complications encountered in both groups. It found that no serious adverse events occurred in either group, but found 25 in the CLE group and 50 in the standard care group to have at least one complication. Complication relating to the surgery included $2(1.0 \%)$ posterior capsule ruptures, $2(1.0 \%)$ iris prolapse, $1(0.5 \%)$ vitreous loss and $1(0.5 \%)$ broken haptic. Three patients in the CLE group required additional surgery, the first, a zonulohyaloido-vitrectomy for aqueous misdirection, the second, a repositioning of a subluxed IOL and the third, anti-VEGF for macula oedema. The rate of posterior capsular rupture, a known risk of CLE surgery was similar to that of large cataract studies. The number of participants with irreversible vision loss was similar in both groups. The study has demonstrating that CLE is safe in PACG even though the technical aspect of surgery is more challenging, it can be safely performed by surgeons experienced in this type of case [65].

The EAGLE study has provided us with high-level evidence to suggest that, for patients who fit their inclusion criteria, CLE should be offered first-line treatment. However, longer term follow-up with clinical data for visual fields and disease progression favouring CLE, will help cement its use in policy. The fact the majority of hospitals that took part in the study are within the UK supports the generalisability of the results to the UK population, important for commissioning treatments. In a time when the NHS has limited funding it may be difficult to commission a treatment that is, in the short term, more expensive that current treatment, although the benefits of reduced medication and follow-up, as well as the quality of life benefits and reduced need for further surgeries could save money in the future. Commissioners will have to weigh the added benefit against the cost. It is worth noting that a glaucoma diagnosis increases the chance of cataract. It could be argued that some patients who receive standard care of LPI and medication will inevitably require lens extraction [70]. In this scenario, the cost-effectiveness of early CLE becomes even more significant. Care commissioning groups already fund lens exchange purely for the purpose of treating glaucoma, however if it is adopted as first-line, the number and overall costs will increase. Service capacity may also be a barrier to the implementation of CLE as a primary treatment option. As LPI is so widely practiced, it will require guidelines from institutions such as the Royal College of Ophthalmologists and the National Institute for health and Care Excellence (NICE) to endorse CLE as a primary treatment to see its widespread adoption in the UK.

\section{Current management of acute angle-closure}

The immediate management of acute primary angle-closure (APAC) is to relieve the symptoms, through reduction of the IOP and reversal of angle-closure [71]. The IOP is usually lowered with medication including oral or topical anhydrase inhibitors, topical beta blockers and topical alpha-2 adrenergic agonists, which all act to reduce the production of aqueous humour. If the angle-closure is thought to be caused by pupillary block or plateau iris then a miotic agent such as pilocarpine should be prescribed. If a 
retrolenticular mechanism is suspected, mydriatics are the drug of choice. In the event these treatments fail to lower the IOP, hyper-osmotic agents can also be used, although there are concerns about the risk of volume overload in frail, ill, elderly patients [72].

Anterior chamber paracentesis has been proposed as a first-line adjunct to topical and systemic medication in APAC. It is said to offer immediate symptomatic relief, although not without risk to the lens [73]. It has also been suggested for medically unresponsive cases, helping to lower the IOP and clear corneal oedema [74]. Another procedure that can be deployed in acute angle-closure is laser iridoplasty. In this procedure, slow, large argon laser burns are applied to the peripheral iris causing the iris to contract and move away from the TM [75]. The procedure has been trialled as an adjunct to topical pilocarpine and timolol and compared against systemic acetazolamide + /intravenous mannitol in managing APAC at presentation. The trial suggested that the procedure resulted in a more rapid reduction in IOP than did the systemic acetazolamide over the first hour of treatment, after which there was no difference [76]. The use of iridoplasty as first-line treatment in APAC was popularised in Hong Kong. In the UK, the technique is more often used in unresponsive cases, after 2-4 h of medication. A further treatment used in medically refractory cases of APAC is diode laser cycloablation. This is almost universally successful in controlling raised pressure, following an unsuccessful period of medical therapy and is often deployed for cases unresponsive to laser iridoplasty [77]. Trabeculectomy in acute angle-closure is not advised.

Once IOP is successfully lowered, definitive intervention should occur within $24 \mathrm{~h}$, aiming to maintain an open angle. LPI is first-line intervention, bypassing the pupil block and allowing the pressure gradient between the anterior and posterior chamber to equalise. This in turn eradicates the anterior convexity of the iris, allowing it to move away from the TM, opening the angle [78]. Attempted LPI is viewed as mandatory in all eyes with acute angle-closure and also in the fellow eye, due to the increased risk of developing acute angle-closure in the future [78-80].

Both the need for, and timing of, lens extraction after acute angle closure have been debated. A randomised controlled trial of early lens extraction compared to LPI showed very significant improvements in IOP control in the lens surgery group [60]. Immediate post attack lens extraction has not been widely adopted due to concerns around the risks of technically demanding surgery in inflamed eyes. Nonetheless, it would seem reasonable to extrapolate these findings to surgery after the immediate episode has settled. Others have suggested combining lens extraction with Goniosynechialysis (GSL) to divide any peripheral anterior synechiae [81, 82]. A study published in
2019 by Husain et al. [83] compared phacoemulsification alone to phacoemulsification plus GSL in 78 eyes with PACG. The study found that both interventions significantly lowered the IOP, but that there was no significant difference between the two groups, and that complication rates were equally low. The contribution from the angle surgery, if any, is hard to determine. If the previous measures fail to lower the IOP then treatment is the same as open angle glaucoma, using IOP lowering medication (e.g., prostaglandin analogues) followed by surgery (trabeculectomy or shunt implant).

\section{Conclusion}

The evidence to support CLE for patients with PACG as a primary treatment option is of high quality. CLE offers meaningful benefits such as improved patient reported quality of life, reduced need for glaucoma medication and surgeries, making it an attractive treatment option. The EAGLE study has laid the foundations for CLE to be implemented into the UK, although further research would help secure this. Conversely, it appears that screening and prophylactic treatment for PACS is not viable due to the low incidence of disease and the limited influence of laser on altering the course of the disease, at least in high-risk Chinese patients, the only group for which high-quality evidence exists. The evidence for a move away from prophylactic PI is less secure than that for adopting the use of CLE in established angle-closure disease. From the UK position, the recent LiGHT trial results supporting more widespread use of selective laser trabeculoplasty as initial treatment for primary open-angle glaucoma will inevitably create a need for greater access to ophthalmic laser resources across the UK [84]. The opportunity costs and finite resources in glaucoma management in the UK point towards a need to reassess whether the policy of offering prophylactic PI is worth continuing.

\section{Compliance with ethical standards}

Conflict of interest The authors declare that they have no conflict of interest.

Publisher's note Springer Nature remains neutral with regard to jurisdictional claims in published maps and institutional affiliations.

\section{References}

1. Chan MPY, Broadway DC, Khawaja AP, Yip JLY, GarwayHeath DF, Burr JM, et al. Glaucoma and intraocular pressure in EPIC-Norfolk Eye Study: cross sectional study. BMJ. 2017;358: j3889. 
2. Quigley HA, Broman AT. The number of people with glaucoma worldwide in 2010 and 2020. Br J Ophthalmol. 2006;90:262-7.

3. Tham Y-C, Li X, Wong TY, Quigley HA, Aung T, Cheng C-Y. Global prevalence of glaucoma and projections of glaucoma burden through 2040. Ophthalmology. 2014;121:2081-90.

4. Foster PJ, Johnson GJ. Glaucoma in China: how big is the problem? Br J Ophthalmol. 2001;85:1277-82.

5. Foster PJ, Buhrmann R, Quigley HA, Johnson GJ. The definition and classification of glaucoma in prevalence surveys. Br J Ophthalmol. 2002;86:238-42.

6. Marchini G, Chemello F, Berzaghi D, Zampieri A. New findings in the diagnosis and treatment of primary angle-closure glaucoma. Prog Brain Res. 2015;221:191-212.

7. Schwartz LW, Rodrigues MM, Spaeth GL, Streeten B, Douglas C. Argon laser iridotomy in the treatment of patients with primary angle-closure or pupillary block glaucoma: a clinicopathologic study. Ophthalmology. 1978;85:294-309.

8. Fankhauser F, der Zypen E. Future of the laser in ophthalmology. Trans Ophthalmol Soc U K. 1982;159:159-63.

9. Prum BE, Herndon LW, Moroi SE, Mansberger SL, Stein JD, Lim MC, et al. Primary angle closure preferred practice pattern ${ }^{\circledast}$ guidelines. Ophthalmology. 2016;123:P1-40.

10. Weinreb RN, Friedman DS (eds). Angle closure and angle-closure glaucoma. NL: Kugler Publications; 2006.

11. Greve EL. Primary angle closure glaucoma: extracapsular cataract extraction or filtering procedure? Int Ophthalmol. 1988;12:157-62.

12. Gunning FP, Greve EL. Uncontrolled primary angle closure glaucoma: results of early intercapsular cataract extraction and posterior chamber lens implantation. Int Ophthalmol. 1991;15:237-47.

13. Gunning FP, Greve EL. Lens extraction for uncontrolled angleclosure glaucoma: long-term follow-up. J Cataract Refract Surg. 1998;24:1347-56.

14. Yang $\mathrm{CH}$, Hung PT. Intraocular lens position and anterior chamber angle changes after cataract extraction in eyes with primary angle-closure glaucoma. J Cataract Refract Surg. 1997;23:1109-13.

15. Lai JSM, Tham CCY, Chan JCH. The clinical outcomes of cataract extraction by phacoemulsification in eyes with primary angle-closure glaucoma (PACG) and co-existing cataract: a prospective case series. J Glaucoma. 2006;15:47-52.

16. Jacobi PC, Dietlein TS, Lüke C, Engels B, Krieglstein GK. Primary phacoemulsification and intraocular lens implantation for acute angle-closure glaucoma. Ophthalmology. 2002;109:1597-603.

17. Hu C-C, Lin H-C, Chen C-S, Kuo N-W. Reduction in admissions of patients with acute primary angle closure occurring in conjunction with a rise in cataract surgery in Taiwan. Acta Ophthalmol. 2008:86:440-5.

18. Keenan TDL, Salmon JF, Yeates D, Goldacre M. Trends in rates of primary angle closure glaucoma and cataract surgery in England from 1968 to 2004. J Glaucoma. 2009;18:201-5.

19. Day AC, Foster PJ. Increases in rates of both laser peripheral iridotomy and phacoemulsification have accompanied a fall in acute angle closure rates in the UK. $\mathrm{Br} \mathrm{J}$ Ophthalmol. 2011;95:1339-40.

20. Gillan SN, Wilson PJ, Knight DS, Sanders R. Trends in acute primary angle-closure glaucoma, peripheral iridotomy and cataract surgery in Scotland, 1998-2012. Ophthalmic Epidemiol. 2016;23:1-5.

21. Devereux JG, Foster PJ, Baasanhu J, Uranchimeg D, Lee P-S, Erdenbeleig T, et al. Anterior chamber depth measurement as a screening tool for primary angle-closure glaucoma in an East Asian population. Arch Ophthalmol. 2000;118:257.
22. Foster PJ, Devereux JG, Alsbirk PH, Lee PS, Uranchimeg D, Machin D, et al. Detection of gonioscopically occludable angles and primary angle closure glaucoma by estimation of limbal chamber depth in Asians: modified grading scheme. Br J Ophthalmol. 2000;84:186-92.

23. Alsbirk PH. Primary angle-closure glaucoma. Oculometry, Epidemiol, Genet a high risk Popul Acta Ophthalmol Suppl. 1976;127:5-31.

24. Sheth HG, Goel R, Jain S. UK national survey of prophylactic YAG iridotomy. Eye. 2005;19:981-4.

25. Teikari J, Raivio I, Nurminen M. Incidence of acute glaucoma in Finland from 1973 to 1982. Graefe's Arch Clin Exp Ophthalmol. 1987;225:357-60.

26. Ivanisević M, Erceg M, Smoljanović A, Trosić Z. The incidence and seasonal variations of acute primary angle-closure glaucoma. Coll Antropol. 2002;26:41-5.

27. Bojić L, Mandić Z, Ivanisević M, Bucan K, Kovacević S, Gverović A, et al. Incidence of acute angle-closure glaucoma in Dalmatia, southern Croatia. Croat Med J. 2004;45:279-82.

28. David R, Tessler Z, Yassur Y. Epidemiology of acute angleclosure glaucoma: incidence and seasonal variations. Ophthalmologica. 1985;191:4-7.

29. Chua PY, Day AC, Lai KL, Hall N, Tan LL, Khan K, et al. The incidence of acute angle closure in Scotland: a prospective surveillance study. Br J Ophthalmol. 2017;102:539-43.

30. Wong TY, Foster PJ, Seah SK, Chew PT. Rates of hospital admissions for primary angle closure glaucoma among Chinese, Malays, and Indians in Singapore. $\mathrm{Br} \mathrm{J}$ Ophthalmol. 2000;84:990-2.

31. Seah SKL, Foster PJ, Chew PTK, Jap A, Oen F, Fam HB, et al. Incidence of acute primary angle-closure glaucoma in Singapore. Arch Ophthalmol. 1997;115:1436.

32. Wilensky JT, Kaufman PL, Frohlichstein D, Gieser DK, Kass MA, Ritch R, et al. Follow-up of angle-closure glaucoma suspects. Am J Ophthalmol. 1993;115:338-46.

33. Thomas R, George R, Parikh R, Muliyil J, Jacob A. Five year risk of progression of primary angle closure suspects to primary angle closure: a population based study. $\mathrm{Br} \quad \mathrm{J}$ Ophthalmol. 2003;87:450-4.

34. Foster PJ, Baasanhu J, Alsbirk PH, Munkhbayar D, Uranchimeg D, Johnson GJ. Glaucoma in Mongolia. Arch Ophthalmol. 1996;114:1235.

35. Nolan WP, Foster PJ, Devereux JG, Uranchimeg D, Johnson GJ, Baasanhu J. YAG laser iridotomy treatment for primary angle closure in east Asian eyes. $\mathrm{Br} \mathrm{J}$ Ophthalmol. 2000;84:1255-9.

36. Nolan WP, Baasanhu J, Undraa A, Uranchimeg D, Ganzorig S, Johnson GJ. Screening for primary angle closure in Mongolia: a randomised controlled trial to determine whether screening and prophylactic treatment will reduce the incidence of primary angle closure glaucoma in an east Asian population. Br J Ophthalmol. 2003;87:271-4.

37. Yip JLY, Foster PJ, Uranchimeg D, Javzandulam B, Javzansuren $\mathrm{D}$, Munhzaya $\mathrm{T}$, et al. Randomised controlled trial of screening and prophylactic treatment to prevent primary angle closure glaucoma. Br J Ophthalmol. 2010;94:1472-7.

38. He M, Foster PJ, Ge J, Huang W, Zheng Y, Friedman DS, et al. Prevalence and clinical characteristics of glaucoma in adult Chinese: a population-based study in Liwan district, Guangzhou. Invest Opthalmol Vis Sci. 2006;47:2782.

39. He M, Foster PJ, Ge J, Huang W, Wang D, Friedman DS, et al. Gonioscopy in adult Chinese: The Liwan Eye Study. Invest Opthalmol Vis Sci. 2006;47:4772.

40. He M, Friedman DS, Ge J, Huang W, Jin C, Lee PS, et al. Laser peripheral iridotomy in primary angle-closure suspects: 
biometric and gonioscopic outcomes. Ophthalmology. 2007; $114: 494-500$.

41. He M, Jiang Y, Huang S, Chang DS, Munoz B, Aung T, et al. Laser peripheral iridotomy for the prevention of angle closure: a single-centre, randomised controlled trial. Lancet. 2019;393:1609-18.

42. Wang N, Ouyang J, Zhou W, Lai M, Ye T, Zeng M, et al. Multiple patterns of angle closure mechanisms in primary angle closure glaucoma in Chinese. Zhonghua Yan Ke Za Zhi. 2000;36:46-51.

43. Sun X, Dai Y, Chen Y, Yu D-Y, Cringle SJ, Chen J, et al. Primary angle closure glaucoma: what we know and what we don't know. Prog Retin Eye Res. 2017;57:26-45.

44. He M, Foster PJ, Johnson GJ, Khaw PT. Angle-closure glaucoma in East Asian and European people. Different diseases? Eye. 2006;20:3-12.

45. Saw S-M, Gazzard G, Friedman DS. Interventions for angleclosure glaucoma. Ophthalmology. 2003;110:1869-79.

46. Chen H-Y, Lin C-L, Lai S-W, Kao C-H. Association of selective serotonin reuptake inhibitor use and acute angle-closure glaucoma. J Clin Psychiatry. 2016;77:e692-6.

47. Subak-Sharpe I, Low S, Nolan W, Foster PJ. Pharmacological and environmental factors in primary angle-closure glaucoma. Br Med Bull. 2010;93:125-43.

48. Amerasinghe N, Zhang J, Thalamuthu A, He M, Vithana EN, Viswanathan A, et al. The heritability and sibling risk of angle closure in Asians. Ophthalmology. 2011;118:480-5.

49. Rudkin AK, Gray TL, Awadalla M, Craig JE. Bilateral simultaneous acute angle closure glaucoma precipitated by nonprescription cold and flu medication. Emerg Med Australas. 2010;22:477-9.

50. Barrett V, Jordan T. Angle closure risk from proprietary medicines. Eye. 2001;15:248-9.

51. Lai JS, Liu DT, Tham CC, Li RT, Lam DS. Epidemiology of acute primary angle-closure glaucoma in the Hong Kong Chinese population: prospective study. Hong Kong Med J. 2001;7:118-23.

52. Turnbull AMJ, Smith M, Ramchandani M. Angle-closure glaucoma on long-haul flights. JAMA Ophthalmol. 2014;132:1474.

53. Wolfs RC, Grobbee DE, Hofman A, de Jong PT. Risk of acute angle-closure glaucoma after diagnostic mydriasis in nonselected subjects: the Rotterdam Study. Invest Ophthalmol Vis Sci. 1997;38:2683-7.

54. Liew G, Mitchell P, Wang JJ, Wong TY. Fundoscopy: to dilate or not to dilate? BMJ. 2006;332:3.

55. Mapstone R. Normal response to pilocarpine and phenylephrine. Br J Ophthalmol. 1977;61:510-1.

56. Mapstone R. Dilating dangerous pupils. $\mathrm{Br} \mathrm{J}$ Ophthalmol. 1977;61:517-24.

57. Foster PJ, Oen FT, Machin D, Ng TP, Devereux JG, Johnson GJ, et al. The prevalence of glaucoma in Chinese residents of Singapore: a cross-sectional population survey of the Tanjong Pagar district. Arch Ophthalmol. 2000;118:1105-11.

58. Friedman D, Vedula SS. Lens extraction for chronic angle-closure glaucoma. Cochrane Database Syst Rev. 2006; CD005555.

59. Melese E, Peterson JR, Feldman RM, Baker LA, Bell NP, Chuang $\mathrm{AZ}$, et al. Comparing laser peripheral iridotomy to cataract extraction in narrow angle eyes using anterior segment optical coherence tomography. PLoS ONE. 2016;11:e0162283.

60. Lam DSC, Leung DYL, Tham CCY, Li FCH, Kwong YYY, Chiu $\mathrm{TYH}$, et al. Randomized trial of early phacoemulsification versus peripheral iridotomy to prevent intraocular pressure rise after acute primary angle closure. Ophthalmology. 2008;115:1134-40.

61. Husain R, Gazzard G, Aung T, Chen Y, Padmanabhan V, Oen F, et al. Initial management of acute primary angle closure: a randomized trial comparing phacoemulsification with laser peripheral iridotomy. Ophthalmology. 2012;119:2274-81.
62. Masis M, Mineault PJ, Phan E, Lin SC. The role of phacoemulsification in glaucoma therapy: a systematic review and metaanalysis. Surv Ophthalmol. 2018;63:700-10.

63. Dada T, Rathi A, Angmo D, Agarwal T, Vanathi M, Khokhar SK, et al. Clinical outcomes of clear lens extraction in eyes with primary angle closure. J Cataract Refract Surg. 2015;41:1470-7.

64. Tham CC, Kwong YY, Baig N, Leung DY, Li FC, Lam DS. Phacoemulsification versus trabeculectomy in medically uncontrolled chronic angle-closure glaucoma without cataract. Ophthalmology. 2013;120:62-7

65. Tham CCY, Leung DYL, Kwong YYY, Liang Y, Peng AY, Li $\mathrm{FCH}$, et al. Factors correlating with failure to control intraocular pressure in primary angle-closure glaucoma eyes with coexisting cataract treated by phacoemulsification or combined phacotrabeculectomy. Asia-Pac J Ophthalmol. 2015;4:56-9.

66. Javanbakht M, Azuara-Blanco A, Burr JM, Ramsay C, Cooper D, Cochran C, et al. Early lens extraction with intraocular lens implantation for the treatment of primary angle closure glaucoma: an economic evaluation based on data from the EAGLE trial. BMJ Open. 2017;7:e013254.

67. Powe NR, Schein OD, Gieser SC, Tielsch JM, Luthra R, Javitt J, et al. Synthesis of the literature on visual acuity and complications following cataract extraction with intraocular lens implantation. Arch Ophthalmol. 1994;112:239-52.

68. Shams PN, Foster PJ. Clinical outcomes after lens extraction for visually significant cataract in eyes with primary angle closure. $\mathrm{J}$ Glaucoma. 2011;21:545-50.

69. Day AC, MacLaren RE, Bunce C, Stevens J, Foster P. Outcomes of phacoemulsification and intraocular lens implantation in microphthalmos and nanophthalmos. J Cataract Refract Surg. 2013;39:87-96.

70. Klein BEK, Klein R, Lee KE. Incidence of age-related cataract over a 10-year interval: the Beaver Dam Eye Study. Ophthalmology. 2002;109:2052-7.

71. Shields SR. Managing eye disease in primary care. Part 3. When to refer for ophthalmologic care. Postgrad Med. 2000;108:99-106.

72. Cantor LB. Medical management of glaucoma. In: Basic and clinical science course (BCSC)—Section 10: Glaucoma. SF, USA: Am Acad Ophthalomol; 2005, p. 157-77.

73. Lam DSC, Chua JKH, Tham CCY, Lai JSM. Efficacy and safety of immediate anterior chamber paracentesis in the treatment of acute primary angle-closure glaucoma: a pilot study. Ophthalmology. 2002;109:64-70.

74. Arnavielle S, Creuzot-Garcher C, Bron AM. Anterior chamber paracentesis in patients with acute elevation of intraocular pressure. Graefe's Arch Clin Exp Ophthalmol. 2007;245:345-50.

75. Ritch R, Tham CCY, Lam DSC. Argon laser peripheral iridoplasty (ALPI): an update. Surv Ophthalmol. 2007;52:279-88.

76. Lam DSC, Lai JSM, Tham CCY, Chua JKH, Poon ASY. Argon laser peripheral iridoplasty versus conventional systemic medical therapy in treatment of acute primary angle-closure glaucoma: a prospective, randomized, controlled trial. Ophthalmology. 2002;109:1591-6.

77. Manna A, Foster P, Papadopoulos M, Nolan W. Cyclodiode laser in the treatment of acute angle closure. Eye. 2012;26:742-5.

78. Quigley HA. Long-term follow-up of laser iridotomy. Ophthalmology. 1981;88:218-24.

79. Robin AL, Pollack IP. Argon laser peripheral iridotomies in the treatment of primary angle closure glaucoma: long-term followUp. Arch Ophthalmol. 1982;100:919-23.

80. Choong YF, Irfan S, Menage MJ. Acute angle closure glaucoma: an evaluation of a protocol for acute treatment. Eye. 1999;13:613-6.

81. Harasymowycz PJ, Papamatheakis DG, Ahmed I, Assalian A, Lesk M, Al-Zafiri Y, et al. Phacoemulsification and 
goniosynechialysis in the management of unresponsive primary angle closure. J Glaucoma. 2005;14:186-9.

82. Teekhasaenee C, Ritch R. Combined phacoemulsification and goniosynechialysis for uncontrolled chronic angle-closure glaucoma after acute angle-closure glaucoma. Ophthalmology. 1999;106:669-75.

83. Husain R, Do T, Lai J, Kitnarong N, Nongpiur, Perera S, et al. Efficacy of phacoemulsification alone vs phacoemulsification with goniosynechialysis in patients with primary angle-closure disease: a randomized clinical trial. JAMA Ophthalmol. Published online 11 July, 2019. https://doi.org/10.1001/jamaophthalmol.2019.2493. 84. Gazzard G, Konstantakopoulou E, Garway-Heath D, Garg A, Vickerstaff V, Hunter R, et al. Selective laser trabeculoplasty versus eye drops for first-line treatment of ocular hypertension and glaucoma (LiGHT): a multicentre randomised controlled trial. Lancet. 2019;393:1505-16. 